BIOENGINEERING

\section{Pharmacokinetic modelling using linked organ chips}

A recent study by Donald Ingber and colleagues reports that a new multi-organchip system can be used to predict human pharmacokinetic responses to drugs, including their clearance by the kidney. "Each chip has two channels that are separated by a porous membrane and lined with human cells: organ cells in one channel and capillary endothelial cells in the other," explains Ingber. "We flow a blood substitute medium through the vascular channel and use a robotic system to transfer this fluid drop by drop from one chip to the next. This approach enables us not only to link different organ chips to create a physiologically coupled 'body on chips' but also to take samples from the flow at every point in the system." The chips are also linked by fluid transfer to an arteriovenous reservoir that mimics the systemic circulation.

The researchers used coupled gut, liver and kidney chips to model pharmacokinetic parameters for orally administered nicotine. They infused the drug into the lumen of the gut chip and showed that it was absorbed by this chip, metabolized by the liver chip and cleared by the kidney chip. Moreover, computationally scaled data from this system quantitatively matched nicotine pharmacokinetic parameters reported in clinical studies. Similarly, pharmacodynamic parameters for intravenously administered cisplatin that were predicted using coupled bone marrow, liver and kidney chips were consistent with published patient data.

"Organ chips can aid in drug discovery and development by replacing animal testing and providing more human-relevant results as well as insights into disease mechanisms, drug efficacy and drug toxicity," says Ingber. "The linked organs in our model can predict which drug regimens produce optimal efficacy in the target organ while minimizing toxicity in other organs. As well as leading to more effective drug treatments, our 'body on chips' technology could save time and money by identifying optimal treatment regimens to introduce into early clinical trials."

Ellen F. Carney

ORIGINAL ARTICLE Herland, A. et al. Quantitative prediction of human pharmacokinetic responses to drugs via fluidically coupled vascularized organ chips. Nat. Biomed. Eng. https:// doi.org/10.1038/s41551-019-0498-9 (2020)

\title{
An autoimmune mechanism may underlie most cases of primary MPGN and C3G
}

C3 glomerulopathy (C3G), a type of primary membranoproliferative glomerulonephritis (MPGN), is generally thought to result from mutations in complement genes. However, a new study suggests that autoimmunity rather than a genetic complement disorder is the key mechanism in the majority of patients.

To investigate genetic causes of primary MPGN and C3G in the UK population, Daniel Gale and colleagues analysed wholegenome sequence data from 146 patients and 6,442 control individuals. "Unexpectedly, we found that rare genetic variants in complement genes were not over-represented in the patients, occurring in around $6 \%$ of individuals in both groups," says Gale. "The frequency of rare variants in all other genes was also similar in patients and controls, suggesting that in the UK population, primary MPGN and C3G are seldom explained by a single gene defect."

The researchers did, however, identify a strong association between common variants at the HLA locus and primary MPGN. "One particular HLA type - DQA ${ }^{\star}$ 05:01,
$\mathrm{DQB}^{\star}{ }^{\star} 02: 01$ and $\mathrm{DRB} 1^{\star} 03: 01$ - was significantly more common in patients with primary MPGN and C3G than controls," explains Gale. "This HLA type is also associated with autoimmune diseases such as type 1 diabetes mellitus (T1DM), which probably explains why people with $\mathrm{C} 3 \mathrm{G}$ are more likely to have a family history of T1DM than the general population." The researchers confirmed their finding by analysing the HLA serotypes of 338 patients with primary or secondary MPGN and 15,614 control individuals.

"Our results suggest that complement gene sequencing in individuals with primary MPGN or C3G is less helpful than previously thought," says Gale. “They may also explain anecdotal reports of patients responding well to immunosuppression and suggest that this is a logical therapeutic strategy."

Ellen F. Carney

ORIGINAL ARTICLE Levine, A. P. et al. Large-scale wholegenome sequencing reveals the genetic architecture of primary membranoproliferative $\mathrm{GN}$ and $\mathrm{C} 3$ glomerulopathy. J. Am. Soc. Nephrol. https://doi.org/10.1681/ASN.2019040433 (2020)

\section{CHRONIC KIDNEY DISEASE}

\section{Kidney sphere grafts for tissue regeneration}

Patients with kidney failure can generally only be treated with kidney replacement therapies, such as transplantation or dialysis. Now, Benjamin Dekel and colleagues report that, in mice, 3D-cultured kidney human cell grafts might encourage tissue regeneration.

"Our previous work suggested that the adult kidney constantly renews itself via lineage-restricted clonal cell proliferation," notes Dekel. "We showed that 3D cultures of adult human kidney cells have tissueregenerative capacity and might be used for kidney regeneration and cellular therapy."

The researchers established 3D spheres (nephrospheres; $\mathrm{nSPHs}$ ) derived from 2D cultures of human adult kidney epithelial cells obtained from either healthy or diseased kidneys. Transcriptomic analysis revealed that whereas cells from the initial 2D culture were enriched for genes associated with epithelialmesenchymal transition and cell cycle, nSPH cells initially expressed genes involved in early kidney development, followed by a mesenchymal-epithelial transition gene profile as they began to differentiate into kidney epithelial cells.
The researchers then transplanted nSPHs into immunodeficient mice. "Human $\mathrm{nSPHs}$ restored self-organogenetic properties that were lost in 2D cultures; long-term engraftment depended on the ability of nSPHs to establish tubular structures and renal parenchyma, which enabled prolonged donor-host interactions," explains Dekel. In mouse models of chronic kidney disease, nSPH grafts led to improved creatinine clearance compared with controls. When co-cultured, $\mathrm{nSPH}$ s derived from diseased kidneys inhibited collagen production by human fibroblasts in the absence of cell contact. " $n S P H$ cell therapy may be unique in targeting fibroblasts in a paracrine fashion," adds Dekel. "nSPH grafts also induced transcriptional signatures of proliferation and release from quiescence in recipient mouse kidney cells; further studies should clarify how these mechanisms might contribute to a therapeutic benefit."

Monica Wang

ORIGINAL ARTICLE Harari-Steinberg, O. et al. Ex vivo expanded 3D human kidney spheres engraft long term and repair chronic renal injury in mice. Cell Rep. https://doi.org/ 10.1016/j.celrep.2019.12.047 (2020) 